\title{
Plasmon Character Index: An Accurate and Efficient Metric for Identifying and Quantifying Plasmons in Molecules
}

\author{
James Langford, Xi Xu, Yang Yang*
}

Theoretical Chemistry Institute and Department of Chemistry, University of Wisconsin-Madison 1101 University Avenue, Madison, WI 53706, USA

\footnotetext{
${ }^{*}$ To whom correspondence should be addressed. E-mail: yyang222@wisc.edu
} 


\begin{abstract}
Plasmons, which are collective and coherent oscillations of charge carriers driven by an external field, play an important role in applications such as solar energy harvesting, sensing, and catalysis. Plasmons can be found in bulk and nanomaterials, and in recent years, plasmons have also been identified in molecules and these molecules have been utilized to build plasmonic devices. As molecular plasmons can no longer be described by classical electrodynamics, a description using quantum mechanics is necessary. Many methods have been developed to identify and quantify molecular plasmons based on the properties of plasmonic excitations. However, there is not currently a method that is widely accepted, connects to collectivity and coherence, and is computationally practical. Here we develop a metric to accurately and efficiently identify and quantify plasmons in molecules. A number, which we call plasmon character index (PCI), can be calculated for each electronic excited state and describes the plasmonicity of the excitation. PCI is developed from the collective and coherent excitation picture in orbitals and shows excellent agreement with the predictions from scaled time-dependent density functional theory but is vastly more computationally efficient. Therefore, PCI can be a useful tool in identifying and quantifying plasmons and will inform the rational design of plasmonic molecules and small nanomaterials.
\end{abstract}

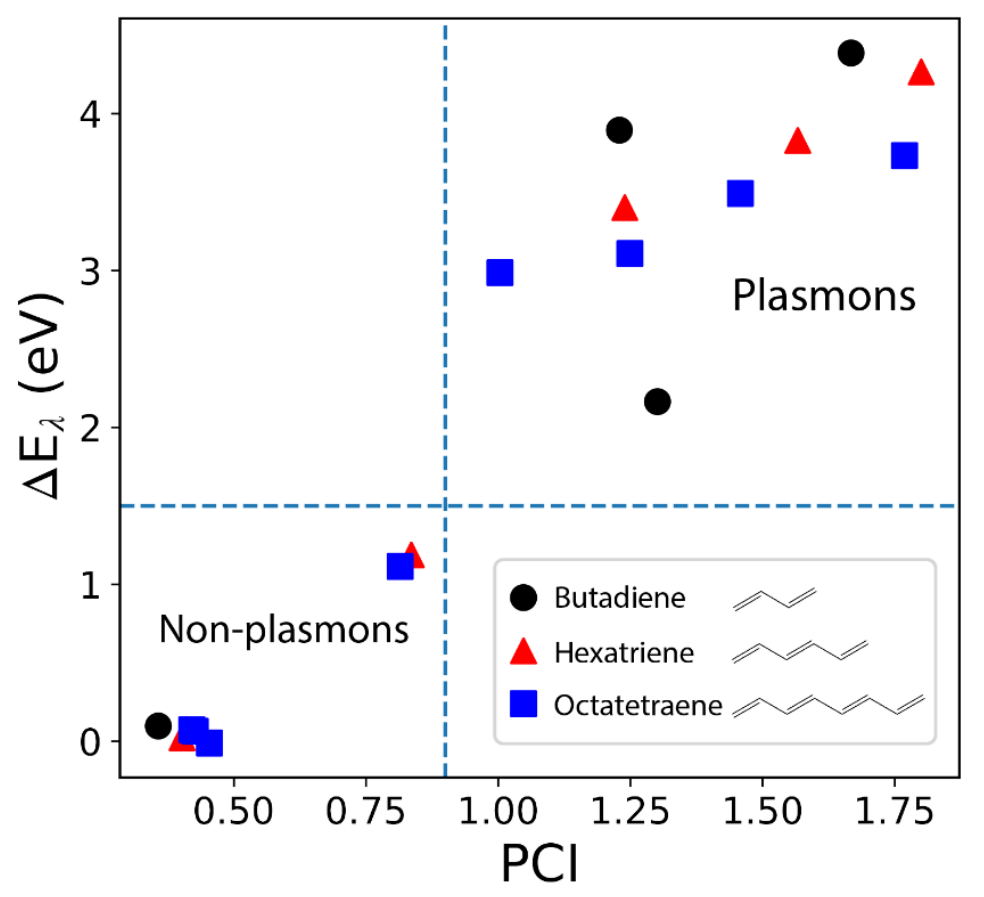


Plasmons are collective and coherent oscillations of charge carriers driven by an external field. ${ }^{1-4}$ They often have large optical absorbances ${ }^{5-7}$ and can capture light at extreme subwavelength dimensions, ${ }^{8-10}$ making them useful in many important applications, such as solar energy harvesting, ${ }^{11-15}$ sensing, ${ }^{16-19}$ and catalysis. ${ }^{20-22}$ Plasmonic materials currently being studied are mostly nanoparticles and nanorods, whose size, shape, material composition, and other features can be easily varied to tune the optical properties of plasmons. ${ }^{6,14,17,23-26}$ Nevertheless, the high degree of accuracy needed for fabrication and lithography places limitations on the practical implementation of these plasmonic materials. ${ }^{14,27,28}$

Recently, plasmons in molecules have been reported by several groups, including in fewatom metal clusters ${ }^{29-33}$ as well as in polycyclic aromatic hydrocarbon ions. ${ }^{27,28,34-38}$ Compared with nanomaterials, these molecules are cheaper to produce and easier to control due to the advantages of chemical synthesis. ${ }^{4,27}$ Furthermore, the length scale of molecules presents new opportunities to capture photons with wavelengths inaccessible to nanoparticles. ${ }^{27,28}$ Thus, significant advances may be achieved with molecular plasmons.

However, the accurate and efficient identification and quantification of plasmons in molecules remain an unsolved problem. ${ }^{7,33,38-45}$ Classical electrodynamics can be used to describe plasmons in bulk materials and large nanoparticles but is not able to describe the behavior of plasmons in molecules or nanoparticles smaller than a few nanometers. ${ }^{33,37,38}$ Therefore, a quantum description for molecular plasmons is necessary. Nevertheless, the connection between a quantum mechanical orbital description and the classical collective and coherent oscillation picture remains unclear. In the past few years, several properties of plasmons have been utilized for identifying plasmons in molecules. One such property is high absorption intensity, which can be quantified with oscillator strength. ${ }^{28,33,40}$ However, high absorption intensity is not equivalent to being plasmonic, and there exist plasmonic excitations with low absorption intensities. ${ }^{41}$ Transition dipole moment additivity has also been used to identify and quantify molecular plasmons based on the inference that it is a measure of coherence. ${ }^{7,26,33,39,46,47}$ By this criterion, the larger the transition dipole moment additivity, the more plasmonic an excitation is. However, it is hard to precisely define this additivity, and with a current definition, ${ }^{33}$ the results may change subject to orbital rotations. Collectivity is another property that has been used to identify molecular plasmons. It has been defined as the number of transitions that significantly contribute to an excitation. ${ }^{38}$ Single particle component analysis (SPCA) $)^{37,38}$ and transition inverse participation 
ratio (TIPR $)^{48}$ have been used to measure collectivity. For each excitation, SPCA directly counts the number of major transitions above a subjectively chosen threshold weight, ${ }^{38}$ whereas TIPR is a more objective numerical index that uses transition density matrix information to measure collectivity ${ }^{48}$ However, both methods measure collectivity but not coherence and thus cannot identify all plasmons. ${ }^{48}$ Plasmonicity Index (PI) ${ }^{44}$ and Generalized Plasmonicity Index (GPI) ${ }^{45}$ identify plasmons based on their large induced potentials from external fields. However, the performance of PI and GPI has only been compared to absorption intensity, ${ }^{44,45}$ which as discussed above is an incomplete metric for plasmonicity. ${ }^{41}$ Excitation lifetimes have also been used to distinguish plasmons from non-plasmons because the vibronic couplings of plasmons substantially reduce their excitation lifetimes relative to non-plasmons. ${ }^{34,37,38,49}$ Nevertheless, this method does not reveal the underlying nature of a plasmon or connect back to the collective and coherent oscillation picture.

Currently, the most theoretically rigorous way to identify plasmons in molecules seems to be scaled time-dependent density functional theory (TDDFT) and scaled wave function theory. ${ }^{41-}$ ${ }^{43}$ These methods identify plasmons based on the differing response of plasmonic and singleparticle excitations to the electron-electron interaction. ${ }^{42} \mathrm{~A}$ scale factor $\lambda$ varying from 0 to 1 is placed in front of the coulomb integral in the post self-consistent-field excited state calculations, and excitation energies are calculated at each scale factor. Plasmonic excitations are sensitive to the change of the scale factor while non-plasmonic excitations are insensitive to scaling. Scaled TDDFT and scaled wavefunction theory connect to the collective and coherent picture of orbital transitions and have been applied to identify plasmons in molecules. ${ }^{41-43,50}$ However, a significant limitation of these scaling methods is that they are computationally expensive as calculations must be performed at many different scale factors from 0 to 1 . An additional challenge is that the energetic evolution of each excited state becomes difficult to trace as the scale factor $\lambda$ varies. This problem is particularly severe in larger and more complex molecules when many excited states are close in energy. ${ }^{33}$

In this work, we introduce plasmon character index (PCI), a metric developed from the collective and coherent picture of orbital transitions to identify and quantify plasmons in molecules. PCI is in excellent agreement with scaled TDDFT but has a dramatically lower computational cost with calculations only performed on the real system (scale factor $\lambda=1$ ). Thus, PCI is an accurate and efficient metric for identifying and quantifying plasmons in molecules. 


\section{$\underline{\text { Theory }}$}

Collective and coherent picture of plasmons in molecules. In the uniform electron gas model, which can be used to understand plasmons in bulk, plasmons are described as coherent superpositions of transitions with equal momentum transfer. ${ }^{1,41-43,50}$ In molecules such as polyenes, conjugated $\pi$ systems can be approximated as uniform electron gases, in which transitions with the same change in orbital quantum number $(\Delta n)$ roughly have the same momentum transfer. ${ }^{42,43} \mathrm{~A}$ constructive addition of these transitions describes collectivity and coherence and therefore results in plasmonic excitations. ${ }^{42,43,50}$ For example, in 1,3-butadiene, a plasmon will occur with $\Delta n=2$ as shown in Figure 1. According to a TDDFT calculation with the B3LYP exchange-correlation functional, ${ }^{51-53}$ it is the $2{ }^{1} \mathrm{Ag}$ excitation with a constructive addition of the $\mathrm{HOMO} \rightarrow \mathrm{LUMO}+1$ and HOMO- $1 \rightarrow$ LUMO transitions, whose transition coefficients are both positive and 0.53 and 0.45 , respectively. This plasmonic behavior can be verified by a scaled TDDFT calculation, in which the excitation energy of this state increases dramatically with the increase of the scaling factor $\lambda$. If we calculate the energy difference between scale factor $\lambda=0$ and scale factor $\lambda=1($ $\Delta E_{\lambda}$ ) for this excitation, the value is $4.39 \mathrm{eV}$ as shown in Table 1. This is in great contrast to the other excitation with $\Delta n=2\left(1^{1} \mathrm{Ag}_{\mathrm{g}}\right)$, whose configurations add destructively and whose corresponding $\Delta E_{\lambda}$ value is a much smaller $0.10 \mathrm{eV}$.

Note that constructive behavior is not necessarily directly observable from inspection of the TDDFT eigenvector, since a change in the phase of an orbital involved will lead to a change in the sign of the eigenvector. ${ }^{42,43}$ Rather, this constructive behavior should be observed by making every molecular orbital have the same phase at a chosen atomic site and correcting the eigenvector signs accordingly. This correction has been done for the transition coefficients in Figure 1 and Table 1. 


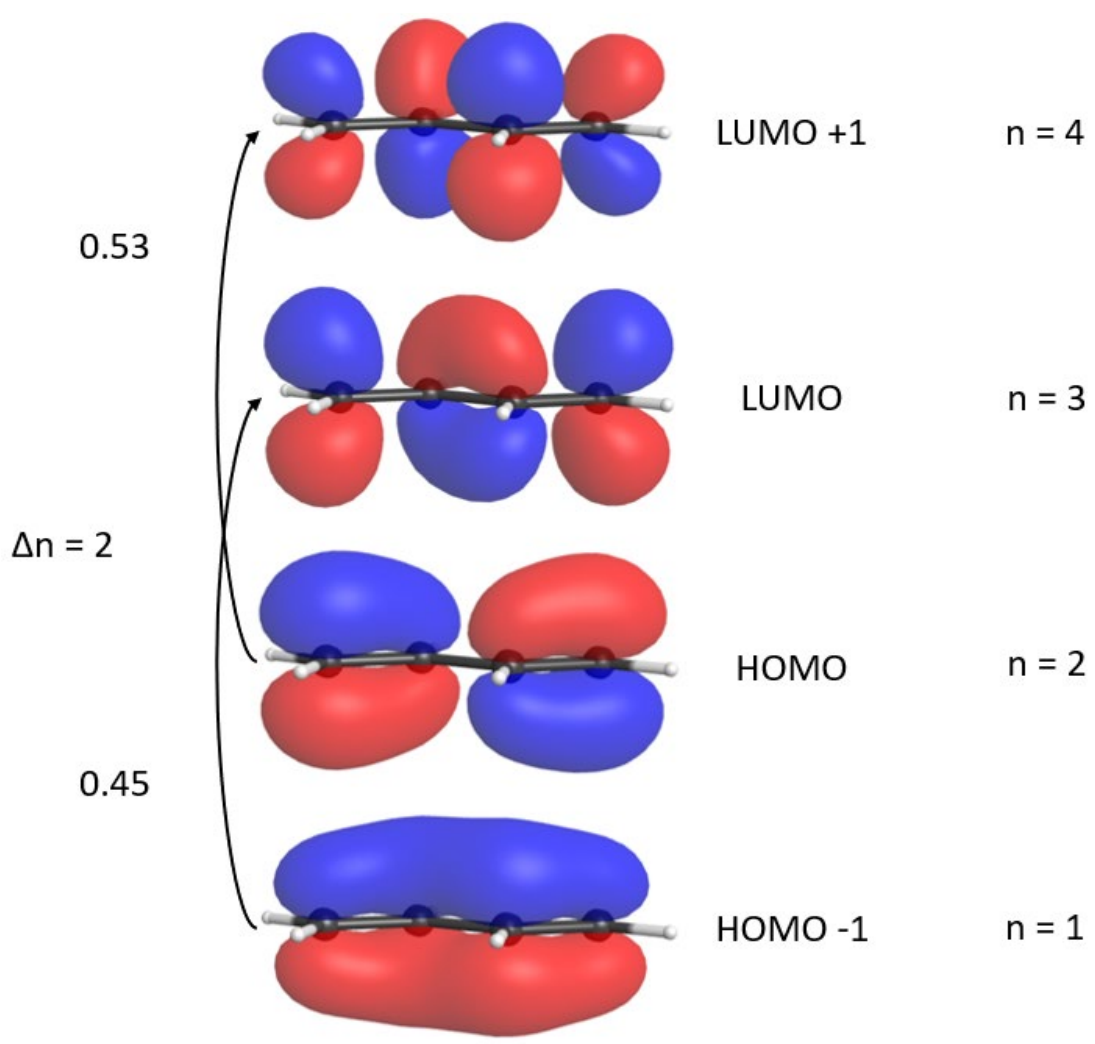

Figure 1. Plasmonic $2{ }^{1} \mathrm{Ag}$ excitation of 1,3-butadiene. It is primarily composed of $\mathrm{HOMO} \rightarrow \mathrm{LUMO}+1$ and HOMO$-1 \rightarrow$ LUMO transitions with $\Delta n=2$. The two transitions add constructively with positive transition coefficients of 0.53 and 0.45 , respectively.

Table 1: $\pi \rightarrow \pi^{*}{ }^{1} \mathrm{Ag}$ Excitations of 1,3-butadiene

\begin{tabular}{cccccc}
\hline Excitation & $\begin{array}{c}\text { Excitation } \\
\text { Energy } \\
(\mathrm{eV})\end{array}$ & $\Delta \mathrm{E}_{\lambda}(\mathrm{eV})$ & Transition & $\Delta \mathrm{n}$ & $\begin{array}{c}\text { TDDFT } \\
\text { transition } \\
\text { coefficients }^{\mathrm{a}}\end{array}$ \\
\hline \multirow{2}{*}{$1{ }^{1} \mathrm{~A} \mathrm{~g}$} & 7.19 & 0.10 & HOMO $-1 \rightarrow$ LUMO & 2 & 0.54 \\
& & & HOMO $\rightarrow$ LUMO +1 & 2 & -0.46 \\
$2{ }^{1} \mathrm{Ag}$ & 8.88 & 4.39 & HOMO $-1 \rightarrow$ LUMO & 2 & 0.45 \\
& & & HOMO $\rightarrow$ LUMO +1 & 2 & 0.53
\end{tabular}

a. Signs of the transition coefficients have been corrected by taking into account the phase of orbitals at a chosen atomic site. 
Development of PCI. Based on the analysis above, we can use the expression

$$
\left|\sum_{i a} X^{i a} A_{R}^{i} A_{R}^{a}\right|
$$

to measure how constructive an excitation is, with $i$ referring to the occupied orbital, $a$ referring to the unoccupied orbital, $R$ referring to an individual atomic site under inspection, $X^{i a}$ referring to the transition coefficient of the $i \rightarrow a$ transition, and $A_{R}$ referring to the phase (-1 or 1$)$ of the top face of the $\pi$ orbital at atomic site $R$. If the signs for every $X^{i a} A_{R}^{i} A_{R}^{a}$ term in the summation are either all positive or all negative, there is a large collectivity and coherence from the different transitions, which is reflected by a large absolute value for this summation.

This expression works well for polyenes, for which the value of $\left|\sum_{i a} X^{i a} A_{R}^{i} A_{R}^{a}\right|$ is the same for all $\mathrm{R}$, that is, regardless of the choice of the atomic site. However, for more general and more complicated $\pi$ systems such as polycyclic aromatic hydrocarbons, the choice of the atomic site could affect the value of $\left|\sum_{i a} X^{i a} A_{R}^{i} A_{R}^{a}\right|$. Nevertheless, the collective and coherent picture of orbital transitions can be recovered by viewing each atomic site as sampling a position in the molecule, and then evaluating the additivity by summing over all atomic sites:

$$
\sum_{R}\left|\sum_{i a} X^{i a} A_{R}^{i} A_{R}^{a}\right|
$$

Further improvements to the expression can be made by replacing the binary sign assignment with the molecular orbital values:

$$
\sum_{R}\left|\sum_{i a} X^{i a} \phi_{i}(R) \phi_{a}(R)\right|
$$

with $\phi_{i}(R)$ being the amplitude of the occupied molecular orbital at atomic site $R$ and $\phi_{a}(R)$ being the amplitude of the virtual molecular orbital at atomic site $R$, both of which are evaluated at the same point above or below the molecular plane. With this change, transitions are weighted differently at each atomic site, and the weights are determined by the value of the molecular orbitals at that site.

Finally, we can extend the sampling to the whole coordinate space instead of limiting the sampling to atomic site positions. This can be achieved by integration over the real space, and we define the final expression as the plasmon character index (PCI): 


$$
\mathrm{PCI}=\int\left|\sum_{i a} X^{i a} \phi_{i}(\mathbf{r}) \phi_{a}(\mathbf{r})\right| d \mathbf{r}
$$

Note that this expression can be further written in terms of transition density within the TammDancoff approximation, which only considers transitions from occupied orbitals to virtual orbitals $^{54}$

$$
\mathrm{PCI}=\int\left|\left\langle\Psi^{*}\left|\Psi^{\dagger}(\mathbf{r}) \Psi(\mathbf{r})\right| \Psi^{0}\right\rangle\right| d \mathbf{r}
$$

Here $\left|\Psi^{0}\right\rangle$ is the ground state, $\left|\Psi^{*}\right\rangle$ is the excited state, and $\Psi^{\dagger}(\mathbf{r})$ and $\Psi$ (r) are field operators that create or annihilate an electron at position $\mathbf{r}$. This expression shows that PCI can be evaluated by integrating the absolute value of the transition density over the whole coordinate space. Because transition density is a property of an excitation and is invariant to orbital rotations, PCI also does not change with any orbital rotations, which is a highly desirable property.

Furthermore, this PCI formulation is universal and is not restricted to $\pi \rightarrow \pi^{*}$ excitations. It can also be evaluated for other types of excitations, such as $\sigma \rightarrow \sigma^{*}, \sigma \rightarrow \pi^{*}$, and $\pi \rightarrow \sigma^{*}$ excitations. We will show $\sigma \rightarrow \pi^{*}$ and $\pi \rightarrow \sigma^{*}$ results later in the paper.

Comparison between PCI and oscillator strength. When we compare PCI with oscillator strength, which is defined as

$$
\begin{aligned}
f_{0 n} & =\frac{2}{3}\left(E_{n}-E_{0}\right)\left|\left\langle\Psi^{0}|\hat{\mathbf{r}}| \Psi^{n}\right\rangle\right|^{2} \\
& =\frac{2}{3}\left(E_{n}-E_{0}\right)\left|\int \mathbf{r}\left\langle\Psi^{0}\left|\Psi^{\dagger}(\mathbf{r}) \Psi(\mathbf{r})\right| \Psi^{n}\right\rangle d \mathbf{r}\right|^{2}
\end{aligned}
$$

we can observe a few key differences. First, the position operator $\hat{\mathbf{r}}$ is in the integrand for oscillator strength but is not present in PCI. Second, the absolute value is taken after integration in oscillator strength whereas it is taken before integration in PCI. In fact, if otherwise, PCI will constantly be zero due to the orbital orthogonalization properties in Eq. (4). Third, oscillator strength has excitation energy $\left(E_{n}-E_{0}\right)$ as a prefactor, whereas PCI focuses only on the collective and coherent picture of the excitation and does not include excitation energy in its expression.

\section{$\underline{\text { Results and Discussion }}$}

Plasmons in polyenes. Plasmons have been previously identified in the $\pi \rightarrow \pi^{*}$ excitations of conjugated polyenes based on the 1D uniform electron gas model. ${ }^{42,43}$ Scaled TDDFT characterizes these plasmonic excitations as being sensitive to the change of the scale factor $\lambda_{.}{ }^{4-}$ 
43 We plot PCI and $\Delta E_{\lambda}$ values together for the lowest 4,6 , and $8 \pi \rightarrow \pi^{*}$ excitations for 1,3 butadiene, 1,3,5-hexatriene, and 1,3,5,7-octatetraene, respectively, in Figure 2. From the figure, we can see a strong correlation between PCI and $\Delta E_{\lambda}$. The larger PCI is, the larger $\Delta E_{\lambda}$ is. This is generally true for all observed $\pi \rightarrow \pi^{*}$ excitations of conjugated diene, triene, and tetraene. Roughly, points with PCI values larger than 0.9 and $\Delta E_{\lambda}$ values larger than $1.5 \mathrm{eV}$ correspond to plasmons identified in previous literature. ${ }^{42}$ Thus, PCI and $\Delta E_{\lambda}$ can both be metrics for plasmonicity in these systems. We will refer to this PCI $=0.9$ and $\Delta E_{\lambda}=1.5 \mathrm{eV}$ as the empirical plasmonicity threshold and analyze all systems in this study with this threshold. Interestingly, although the conjugated $\pi$ systems are of different sizes, the $\pi \rightarrow \pi^{*}$ excitations in these three systems all roughly follow the same trend, which we expect will also hold for longer conjugated polyene systems.

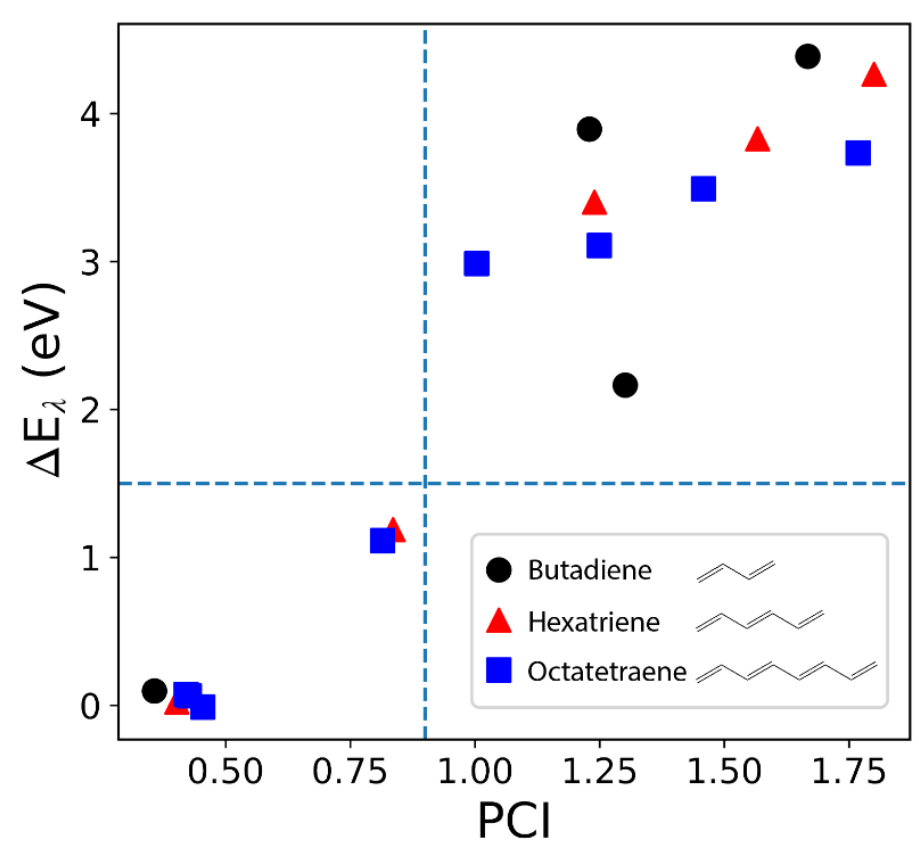

Figure 2. PCI and $\Delta E_{\lambda}$ for $\pi \rightarrow \pi^{*}$ excitations of conjugated polyenes. The lowest 4 , 6 , and 8 excitations are plotted for 1,3-butadiene, 1,3,5-hexatriene, and 1,3,5,7-octatetraene, respectively. PCI and $\Delta E_{\lambda}$ have a strong correlation with all data points roughly following the same trend.

Plasmons in polyacenes. Plasmonic excitations also exist in polyacenes. ${ }^{28,36,39,55}$ In principle, the plasmonicity of $\pi \rightarrow \pi^{*}$ excitations in polyacenes can be understood using a $2 \mathrm{D}$ 
uniform electron gas model. ${ }^{42}$ However, the hexagonal shape of the benzene rings brings complication to the analysis and makes it difficult to assign quantum numbers for momentum transfer. Thus, without referring to the momentum transfer picture, we simply compare PCI with $\Delta E_{\lambda}$ for the lowest 4,6 , and $8 \pi \rightarrow \pi^{*}$ excitations for naphthalene, anthracene, and tetracene, respectively. The results are shown in Figure 3.

Similar to the $\pi \rightarrow \pi^{*}$ excitations of polyenes, we can again observe a strong correlation between PCI and $\Delta E_{\lambda}$ in the $\pi \rightarrow \pi^{*}$ excitations of polyacenes. In contrast to polyenes, we can directly observe two clusters among the lowest $\pi \rightarrow \pi^{*}$ excitations in polyacenes. The cluster with small PCI and small $\Delta \mathrm{E}_{\lambda}$ contains non-plasmonic excitations, whereas the other cluster with large PCI and large $\Delta \mathrm{E}_{\lambda}$ contains plasmonic excitations. We find that the empirical plasmonicity threshold can also be applied to polyacenes, with PCI values larger than 0.9 and $\Delta E_{\lambda}$ values larger than $1.5 \mathrm{eV}$ corresponding to plasmonic excitations.

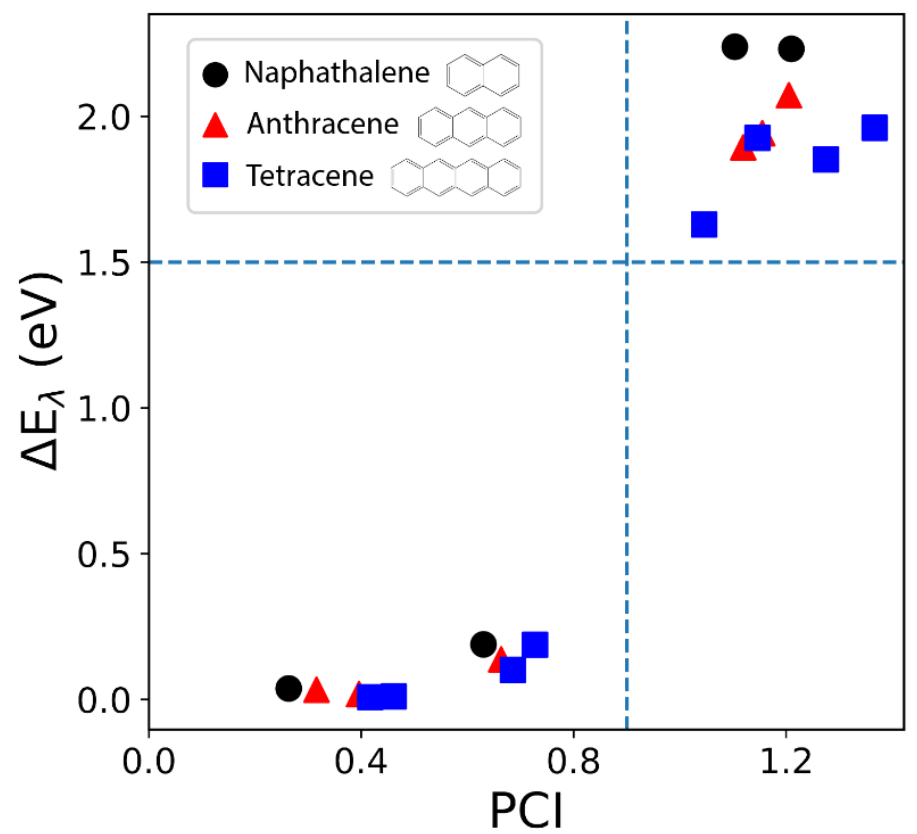

Figure 3. PCI and $\Delta E_{\lambda}$ for $\pi \rightarrow \pi^{*}$ excitations of polyacenes. The lowest 4,6 , and 8 excitations are plotted for naphthalene, anthracene, and tetracene, respectively. Two clusters can be identified with plasmons having larger PCI and larger $\Delta E_{\lambda}$ and non-plasmons having smaller PCI and smaller $\Delta E_{\lambda}$. 
Plasmons for charged systems. Plasmonic excitations for charged states have been investigated experimentally in prior literature. ${ }^{27,28,35,37,38}$ Here we compute and plot PCI and $\Delta \mathrm{E}_{\lambda}$ for the lowest $5 \pi \rightarrow \pi^{*}$ excitations in the neutral, cation, and anion states of anthracene in Figure 4.

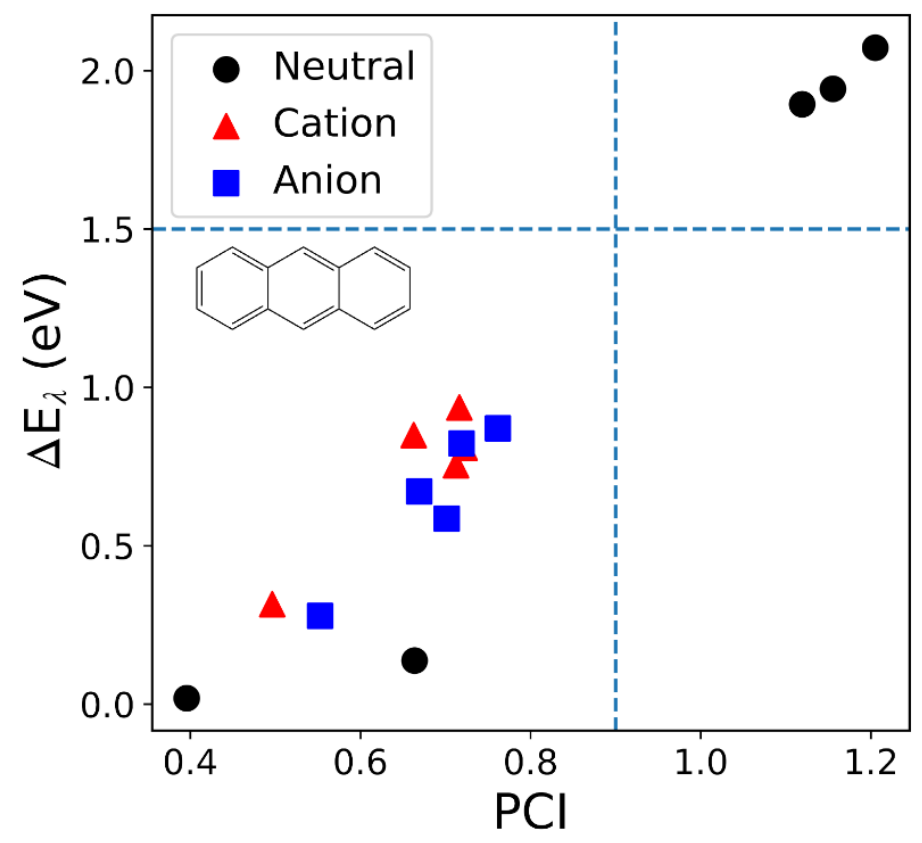

Figure 4. PCI and $\Delta E_{\lambda}$ for the lowest $5 \pi \rightarrow \pi^{*}$ excitations in neutral, cation, and anion states of anthracene. The strong correlation between PCI and $\Delta E_{\lambda}$ also holds for charged states. The lowest few excitations of charged states are related to the SOMO. They have moderate PCI values and can be categorized as semi-plasmons.

Similar to the neutral system, $\pi \rightarrow \pi^{*}$ excitations in the cation and anion of anthracene also show a strong correlation between PCI and $\Delta E_{\lambda}$ and follow the same trend as the neutral system. However, unlike the neutral system with two distinct clusters, the lowest $\pi \rightarrow \pi^{*}$ excitations in the cation and anion form one cluster in the middle. The common feature of these low-lying excitations is that they all involve transitions into or out of the singly occupied molecular orbital (SOMO). They appear in the visible range and have moderate PCI values in between the PCI values for the plasmonic and non-plasmonic excitations of the neutral system. If we apply the empirical plasmonicity threshold ( $\mathrm{PCI}>0.9, \Delta E_{\lambda}>1.5 \mathrm{eV}$ ) to these charged systems, these excitations do not quite meet the criteria of being plasmonic. However, their plasmonicity is generally larger than 
those of the neutral non-plasmons. Thus, we may refer to these excitations as semi-plasmons. Hence, changing from the neutral state to the cation or anion state turns on semi-plasmons in the visible region for polycyclic aromatic hydrocarbons. This is largely in agreement with prior literature that has observed the same effect experimentally and rationalized the results based on changes in the electronic structure of the molecule. ${ }^{27,28,35,37,38}$ Nevertheless, one important result from our study is that these SOMO-related excitations are less plasmonic than the plasmons in neutral systems, which is reflected by the moderate PCI and $\Delta E_{\lambda}$ values.

Plasmons in neutral and charged perylene. We next test PCI in larger and more practical polycyclic aromatic hydrocarbon systems to verify its generality. We use perylene and its charged states as test systems because they have been used to make plasmonic devices in recent literature. ${ }^{35,38} \mathrm{PCI}$ and $\Delta E_{\lambda}$ values for the lowest $5 \pi \rightarrow \pi^{*}$ excitations in the neutral, cation, and anion states of perylene are plotted in Figure 5. We again see a strong correlation between PCI and $\Delta E_{\lambda}$, which we also observed in all systems studied above where both are reliable metrics for plasmonicity. This indicates that PCI and $\Delta E_{\lambda}$ continue to be reliable metrics for identifying and quantifying plasmons in large systems. According to the empirical plasmonicity threshold, there is one plasmon in the neutral system, with a PCI value of 1.25 and a $\Delta E_{\lambda}$ value of $1.74 \mathrm{eV}$, which is dominated by the HOMO to LUMO transition. For the charged states, the lowest few $\pi \rightarrow \pi^{*}$ excitations all involve the SOMO, as was the case with the charged states of anthracene. However, for the excitation in both the anion and cation with the largest PCI and $\Delta E_{\lambda}$ values, its largest transition is still from what was the HOMO in the neutral state to what was the LUMO in the neutral state. These two excitations appear slightly below the empirical plasmonicity threshold and thus may be described as semi-plasmons. They have relatively large oscillator strengths and occur within the visible range, matching prior experiments in which the differences in spectra between the neutral and charged states of perylene have been utilized to make a color switching electrochromic device with applied voltage. ${ }^{35,38}$ 


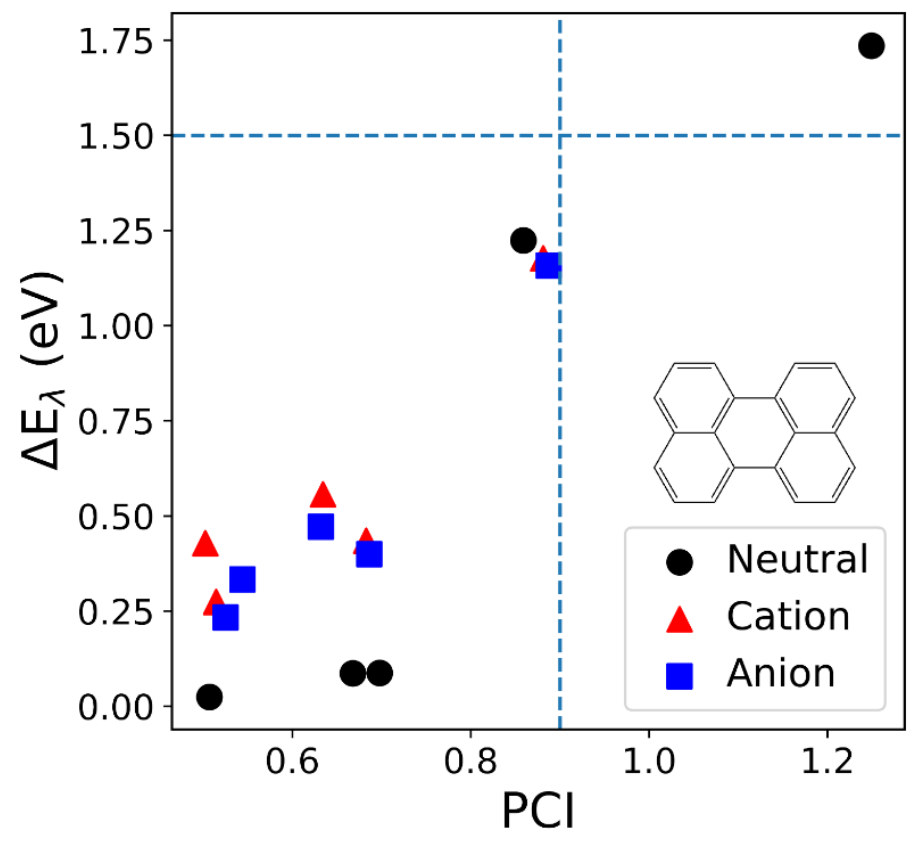

Figure 5. PCI and $\Delta E_{\lambda}$ for the lowest $5 \pi \rightarrow \pi^{*}$ excitations in neutral, cation, and anion states of perylene. The strong correlation between PCI and $\Delta E_{\lambda}$ can still be observed in this more complex molecule.

Plasmons for other types of excitations. We also tested PCI on excitations that are not of the $\pi \rightarrow \pi^{*}$ type. The results of the lowest $10 \sigma \rightarrow \pi^{*}$ and $\pi \rightarrow \sigma^{*}$ excitations and lowest $6 \pi \rightarrow \pi^{*}$ excitations for 1,3,5-hexatriene are plotted in Figure 6. We see a strong correlation between PCI and $\Delta E_{\lambda}$ for the $\sigma \rightarrow \pi^{*}$ and $\pi \rightarrow \sigma^{*}$ excitations as well as for the $\pi \rightarrow \pi^{*}$ excitations. This indicates that while the collective and coherent picture of orbital transitions in the $\pi \rightarrow \pi^{*}$ excitations of polyenes is the starting point for the development of PCI, PCI is also able to identify and quantify plasmons regardless of the type of excitation and thus is a general tool to describe plasmonicity. Notably, the $\pi \rightarrow \pi^{*}$ excitations follow a different trend than the $\sigma \rightarrow \pi^{*}$ and $\pi \rightarrow \sigma^{*}$ excitations, indicating that the $\Delta E_{\lambda}$-PCI correlation depends on the type of excitation. According to the empirical plasmonicity threshold, most of these $\sigma \rightarrow \pi^{*}$ and $\pi \rightarrow \sigma^{*}$ excitations belong to the nonplasmon category, which is reasonable since it is difficult for electrons in different types of bonds to oscillate collectively and coherently. 


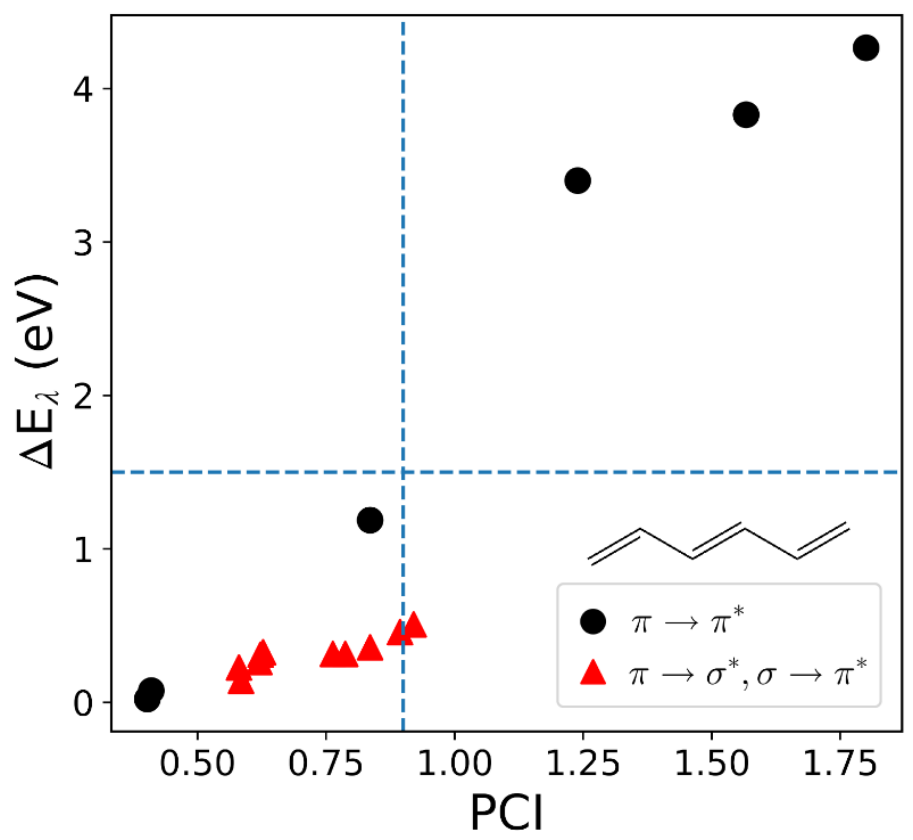

Figure 6. PCI and $\Delta E_{\lambda}$ for $\pi \rightarrow \pi^{*}, \sigma \rightarrow \pi^{*}$ and $\pi \rightarrow \sigma^{*}$ excitations of 1,3,5-hexatriene. The strong correlation between PCI and $\Delta E_{\lambda}$ still can be observed in $\sigma \rightarrow \pi^{*}$ and $\pi \rightarrow \sigma^{*}$ excitations, indicating the generality of PCI. Notably, the $\sigma \rightarrow \pi^{*}$ and $\pi \rightarrow \sigma^{*}$ excitations are generally less plasmonic and follow a different trend than the $\pi \rightarrow \pi^{*}$ excitations.

PCI and oscillator strength. As noted previously, oscillator strength is an incomplete metric for plasmonicity. In Figure 7, the relationship between oscillator strength and PCI as well as the relationship between oscillator strength and $\Delta E_{\lambda}$ are plotted for the low-lying $\pi \rightarrow \pi^{*}$ excitations of conjugated polyenes (1,3-butadiene, 1,3,5-hexatriene, and 1,3,5,7-octatetraene). In contrast to the plot of $\Delta E_{\lambda}$ and PCI (Figure 2) for these same molecules where a strong trend is observed, there is no apparent correlation between oscillator strength and PCI or between oscillator strength and $\Delta \mathrm{E}_{\lambda}$. Furthermore, some plasmonic excitations have small oscillator strength, thus cannot be identified with oscillator strength alone. Hence, PCI and $\Delta E_{\lambda}$ have much stronger predictive power than oscillator strength in identifying and quantifying plasmonic excitations, but between PCI and $\Delta E_{\lambda}$, PCI is vastly more efficient. 

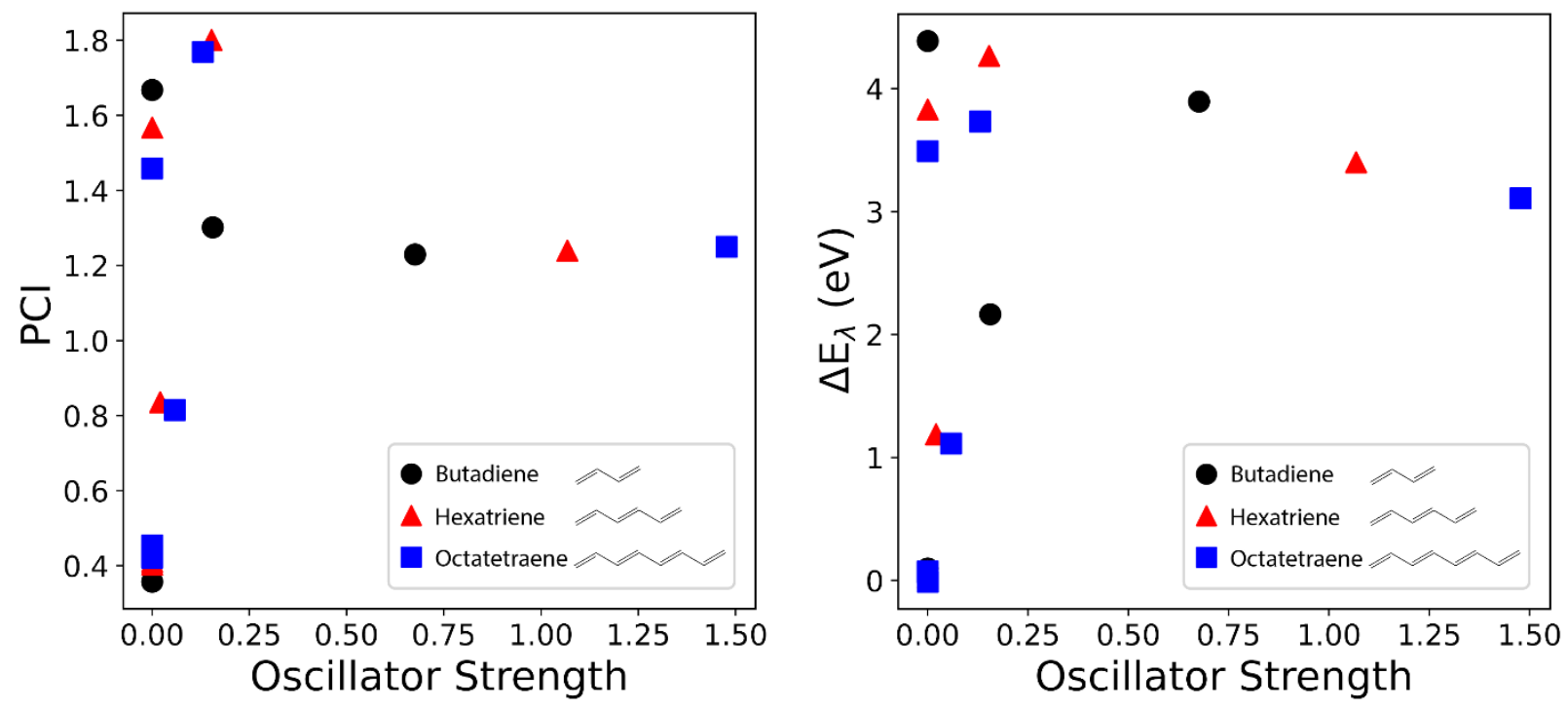

Figure 7. Oscillator strength and PCI as well as oscillator strength and $\Delta \mathrm{E}_{\lambda}$ for $\pi \rightarrow \pi^{*}$ excitations of conjugated polyenes. The lowest 4,6 , and 8 excitations are plotted for 1,3-butadiene, 1,3,5hexatriene, and 1,3,5,7-octatetraene, respectively. There is no apparent correlation between oscillator strength and $\Delta E_{\lambda}$, suggesting that oscillator strength is not a good metric for plasmonicity.

\section{Conclusions}

In this paper, we developed PCI, a metric to accurately and efficiently identify and quantify plasmons in molecules. PCI evaluates the collectivity and coherence of excitations in the orbital picture using transition density information. PCI is in excellent agreement with scaled TDDFT results in identifying and quantifying plasmons in conjugated polyenes, polyacenes, and more complex polycyclic aromatic hydrocarbons. It can be applied to both neutral and charged systems and works generally regardless of excitation type. Compared to scaled TDDFT, PCI does not need to artificially scale the Coulomb interactions or trace the evolution of excitations. It only performs calculations on the real system and is thus vastly computationally cheaper than scaled TDDFT, making PCI practical to implement for large and complex molecules. We expect PCI to be extendable to other quantum systems such as small nanomaterials. Thus, PCI is a powerful tool for efficiently identifying and quantifying plasmonic excitations regardless of size or excitation type and can facilitate the rational design of plasmonics in molecules and nanomaterials. 


\section{$\underline{\text { Methods }}$}

We implemented both scaled TDDFT and PCI in an in-house version of PySCF. ${ }^{56,57} \mathrm{We}$ tested PCI and compared the results with scaled TDDFT on a series of conjugated trans-polyenes (1,3-butadiene, 1,3,5-hexatriene, 1,3,5,7-octatetraene), polyacenes (naphthalene, anthracene, tetracene), and a more complicated polycyclic aromatic hydrocarbon molecule perylene, which has been used to make plasmonic devices in previous literature. ${ }^{35}$ The geometry optimizations as well as all subsequent scaled TDDFT and PCI calculations are performed with the B3LYP functional and 6-31G basis set.

\section{Acknowledgments}

We are grateful for the support and funding from the University of Wisconsin via the Wisconsin Alumni Research Foundation. We are also grateful to Zehua Chen for helpful discussions. 


\section{References}

(1) Pines, D.; Bohm, D. A Collective Description of Electron Interactions: II. Collective vs Individual Particle Aspects of the Interactions. Phys. Rev. 1952, 85 (2), 338-353.

(2) Ozbay, E. Plasmonics: Merging Photonics and Electronics at Nanoscale Dimensions. Science 2006, 311 (5758), 189-193.

(3) Halas, N. J.; Lal, S.; Chang, W.-S.; Link, S.; Nordlander, P. Plasmons in Strongly Coupled Metallic Nanostructures. Chem. Rev. 2011, 111 (6), 3913-3961.

(4) Wilson, A. J.; Willets, K. A. Molecular Plasmonics. Annual Review of Analytical Chemistry 2016, $9(1), 27-43$.

(5) El-Sayed, M. A. Some Interesting Properties of Metals Confined in Time and Nanometer Space of Different Shapes. Acc. Chem. Res. 2001, 34 (4), 257-264.

(6) Burda, C.; Chen, X.; Narayanan, R.; El-Sayed, M. A. Chemistry and Properties of Nanocrystals of Different Shapes. Chem. Rev. 2005, 105 (4), 1025-1102.

(7) Guidez, E. B.; Aikens, C. M. Quantum Mechanical Origin of the Plasmon: From Molecular Systems to Nanoparticles. Nanoscale 2014, 6 (20), 11512-11527.

(8) Li, K.; Stockman, M. I.; Bergman, D. J. Self-Similar Chain of Metal Nanospheres as an Efficient Nanolens. Phys. Rev. Lett. 2003, 91 (22), 227402.

(9) Schuller, J. A.; Barnard, E. S.; Cai, W.; Jun, Y. C.; White, J. S.; Brongersma, M. L. Plasmonics for Extreme Light Concentration and Manipulation. Nature Materials 2010, 9 (3), 193-204.

(10) Brongersma, M. L.; Halas, N. J.; Nordlander, P. Plasmon-Induced Hot Carrier Science and Technology. Nature Nanotech 2015, 10 (1), 25-34.

(11) Atwater, H. A.; Polman, A. Plasmonics for Improved Photovoltaic Devices. Nature Materials 2010, 9 (3), 205-213.

(12) Wu, K.; Chen, J.; McBride, J. R.; Lian, T. Efficient Hot-Electron Transfer by a Plasmon-Induced Interfacial Charge-Transfer Transition. Science 2015, 349 (6248), 632-635.

(13) Li, J.; Cushing, S. K.; Meng, F.; Senty, T. R.; Bristow, A. D.; Wu, N. Plasmon-Induced Resonance Energy Transfer for Solar Energy Conversion. Nature Photonics 2015, 9 (9), 601-607.

(14) Cushing, S. K.; Wu, N. Progress and Perspectives of Plasmon-Enhanced Solar Energy Conversion. J. Phys. Chem. Lett. 2016, 7 (4), 666-675.

(15) Yu, P.; Zhang, F.; Li, Z.; Zhong, Z.; Govorov, A.; Fu, L.; Tan, H.; Jagadish, C.; Wang, Z. Giant Optical Pathlength Enhancement in Plasmonic Thin Film Solar Cells Using Core-Shell Nanoparticles. J. Phys. D: Appl. Phys. 2018, 51 (29), 295106.

(16) Homola, J.; Yee, S. S.; Gauglitz, G. Surface Plasmon Resonance Sensors: Review. Sensors and Actuators B: Chemical 1999, 54 (1), 3-15.

(17) Willets, K. A.; Van Duyne, R. P. Localized Surface Plasmon Resonance Spectroscopy and Sensing. Annual Review of Physical Chemistry 2007, 58 (1), 267-297.

(18) Mayer, K. M.; Hafner, J. H. Localized Surface Plasmon Resonance Sensors. Chem. Rev. 2011, 111 (6), 3828-3857.

(19) Celiksoy, S.; Ye, W.; Wandner, K.; Kaefer, K.; Sönnichsen, C. Intensity-Based Single Particle Plasmon Sensing. Nano Lett. 2021, 21 (5), 2053-2058.

(20) Linic, S.; Christopher, P.; Ingram, D. B. Plasmonic-Metal Nanostructures for Efficient Conversion of Solar to Chemical Energy. Nature Mater 2011, 10 (12), 911-921.

(21) Seemala, B.; Therrien, A. J.; Lou, M.; Li, K.; Finzel, J. P.; Qi, J.; Nordlander, P.; Christopher, P. Plasmon-Mediated Catalytic O2 Dissociation on Ag Nanostructures: Hot Electrons or Near Fields? ACS Energy Lett. 2019, 4 (8), 1803-1809.

(22) Wu, Q.; Zhou, L.; Schatz, G. C.; Zhang, Y.; Guo, H. Mechanistic Insights into Photocatalyzed H2 Dissociation on Au Clusters. J. Am. Chem. Soc. 2020, 142 (30), 13090-13101. 
(23) Franzl, T.; Wilk, T.; von Plessen, G.; Feldmann, J.; Wilson, O.; Mulvaney, P.; Sönnichsen, C. Drastic Reduction of Plasmon Damping in Gold Nanorods. Phys. Rev. Lett. 2002, 88 (7), 077402. Prodan, E.; Radloff, C.; Halas, N. J.; Nordlander, P. A Hybridization Model for the Plasmon Response of Complex Nanostructures. Science 2003, 302 (5644), 419-422.

(25) Atay, T.; Song, J.-H.; Nurmikko, A. V. Strongly Interacting Plasmon Nanoparticle Pairs: From Dipole-Dipole Interaction to Conductively Coupled Regime. Nano Lett. 2004, 4 (9), 1627-1631.

(26) Guidez, E. B.; Aikens, C. M. Diameter Dependence of the Excitation Spectra of Silver and Gold Nanorods. J. Phys. Chem. C 2013, 117 (23), 12325-12336.

(27) Manjavacas, A.; Marchesin, F.; Thongrattanasiri, S.; Koval, P.; Nordlander, P.; Sánchez-Portal, D.; García de Abajo, F. J. Tunable Molecular Plasmons in Polycyclic Aromatic Hydrocarbons. ACS Nano 2013, 7 (4), 3635-3643.

(28) Lauchner, A.; Schlather, A. E.; Manjavacas, A.; Cui, Y.; McClain, M. J.; Stec, G. J.; García de Abajo, F. J.; Nordlander, P.; Halas, N. J. Molecular Plasmonics. Nano Lett. 2015, 15 (9), 62086214.

(29) Aikens, C. M.; Li, S.; Schatz, G. C. From Discrete Electronic States to Plasmons: TDDFT Optical Absorption Properties of Ag $\mathrm{n}(\mathrm{n}=10,20,35,56,84,120)$ Tetrahedral Clusters. J. Phys. Chem. C 2008, 112 (30), 11272-11279.

(30) Bae, G.-T.; Aikens, C. M. Time-Dependent Density Functional Theory Studies of Optical Properties of Ag Nanoparticles: Octahedra, Truncated Octahedra, and Icosahedra. J. Phys. Chem. C 2012, 116 (18), 10356-10367.

(31) Joshi, C. P.; Bootharaju, M. S.; Alhilaly, M. J.; Bakr, O. M. [Ag25(SR)18]-: The "Golden" Silver Nanoparticle. J. Am. Chem. Soc. 2015, 137 (36), 11578-11581.

(32) Chakraborty, I.; Pradeep, T. Atomically Precise Clusters of Noble Metals: Emerging Link between Atoms and Nanoparticles. Chem. Rev. 2017, 117 (12), 8208-8271.

(33) Gieseking, R. L. M.; Ashwell, A. P.; Ratner, M. A.; Schatz, G. C. Analytical Approaches To Identify Plasmon-like Excited States in Bare and Ligand-Protected Metal Nanoclusters. J. Phys. Chem. C 2020, 124 (5), 3260-3269.

(34) Cui, Y.; Lauchner, A.; Manjavacas, A.; García de Abajo, F. J.; Halas, N. J.; Nordlander, P. Molecular Plasmon-Phonon Coupling. Nano Letters 2016, 16 (10), 6390-6395.

(35) Stec, G. J.; Lauchner, A.; Cui, Y.; Nordlander, P.; Halas, N. J. Multicolor Electrochromic Devices Based on Molecular Plasmonics. ACS Nano 2017, 11 (3), 3254-3261.

(36) Zhang, K.; Wang, H.; Fang, M. Electrical Tunability of Molecular Plasmons in Acenes. Chemical Physics Letters 2019, 721, 38-42.

(37) Chapkin, K. D.; Bursi, L.; Stec, G. J.; Lauchner, A.; Hogan, N. J.; Cui, Y.; Nordlander, P.; Halas, N. J. Lifetime Dynamics of Plasmons in the Few-Atom Limit. PNAS 2018, 115 (37), 9134-9139.

(38) Chapkin, K. D.; Bursi, L.; Clark, B. D.; Wu, G.; Lauchner, A.; Tsai, A.-L.; Nordlander, P.; Halas, N. J. Effects of Electronic Structure on Molecular Plasmon Dynamics. J. Phys. Chem. C 2020, 124 (37), 20450-20457.

(39) Guidez, E. B.; Aikens, C. M. Origin and TDDFT Benchmarking of the Plasmon Resonance in Acenes. J. Phys. Chem. C 2013, 117 (41), 21466-21475.

(40) Guidez, E. B.; Aikens, C. M. Plasmon Resonance Analysis with Configuration Interaction. Phys. Chem. Chem. Phys. 2014, 16 (29), 15501-15509.

(41) Bernadotte, S.; Evers, F.; Jacob, C. R. Plasmons in Molecules. The Journal of Physical Chemistry C 2013, 117 (4), 1863-1878.

(42) Krauter, C. M.; Schirmer, J.; Jacob, C. R.; Pernpointner, M.; Dreuw, A. Plasmons in Molecules: Microscopic Characterization Based on Orbital Transitions and Momentum Conservation. $J$. Chem. Phys. 2014, 141 (10), 104101.

(43) Krauter, C. M.; Bernadotte, S.; Jacob, C. R.; Pernpointner, M.; Dreuw, A. Identification of Plasmons in Molecules with Scaled Ab Initio Approaches. J. Phys. Chem. C 2015, 119 (43), 24564-24573. 
(44) Bursi, L.; Calzolari, A.; Corni, S.; Molinari, E. Quantifying the Plasmonic Character of Optical Excitations in Nanostructures. ACS Photonics 2016, 3 (4), 520-525.

(45) Zhang, R.; Bursi, L.; Cox, J. D.; Cui, Y.; Krauter, C. M.; Alabastri, A.; Manjavacas, A.; Calzolari, A.; Corni, S.; Molinari, E.; Carter, E. A.; García de Abajo, F. J.; Zhang, H.; Nordlander, P. How To Identify Plasmons from the Optical Response of Nanostructures. ACS Nano 2017, 11 (7), 7321-7335.

(46) Guidez, E. B.; Aikens, C. M. Theoretical Analysis of the Optical Excitation Spectra of Silver and Gold Nanowires. Nanoscale 2012, 4 (14), 4190-4198.

(47) Gieseking, R. L.; Ratner, M. A.; Schatz, G. C. Semiempirical Modeling of Ag Nanoclusters: New Parameters for Optical Property Studies Enable Determination of Double Excitation Contributions to Plasmonic Excitation. J. Phys. Chem. A 2016, 120 (26), 4542-4549.

(48) Casanova, D.; Matxain, J. M.; Ugalde, J. M. Plasmonic Resonances in the Al13-Cluster: Quantification and Origin of Exciton Collectivity. J. Phys. Chem. C 2016, 120 (23), 12742-12750.

(49) Yi, C.; Dongare, P. D.; Su, M.-N.; Wang, W.; Chakraborty, D.; Wen, F.; Chang, W.-S.; Sader, J. E.; Nordlander, P.; Halas, N. J.; Link, S. Vibrational Coupling in Plasmonic Molecules. Proceedings of the National Academy of Sciences of the United States of America 2017, 114 (44), 11621-11626.

(50) Ring, P.; Schuck, P. The Nuclear Many-Body Problem; Theoretical and Mathematical Physics, The Nuclear Many-Body Problem; Springer-Verlag: Berlin Heidelberg, 1980.

(51) Becke, A. D. Density-Functional Exchange-Energy Approximation with Correct Asymptotic Behavior. Phys. Rev. A 1988, 38 (6), 3098-3100.

(52) Becke, A. D. Density-functional Thermochemistry. III. The Role of Exact Exchange. J. Chem. Phys. 1993, 98 (7), 5648-5652.

(53) Lee, C.; Yang, W.; Parr, R. G. Development of the Colle-Salvetti Correlation-Energy Formula into a Functional of the Electron Density. Phys. Rev. B 1988, 37 (2), 785-789.

(54) Dreuw, A.; Head-Gordon, M. Single-Reference Ab Initio Methods for the Calculation of Excited States of Large Molecules. Chem. Rev. 2005, 105 (11), 4009-4037.

(55) Bursi, L.; Calzolari, A.; Corni, S.; Molinari, E. Light-Induced Field Enhancement in Nanoscale Systems from First-Principles: The Case of Polyacenes. ACS Photonics 2014, 1 (10), 1049-1058.

(56) Sun, Q.; Berkelbach, T. C.; Blunt, N. S.; Booth, G. H.; Guo, S.; Li, Z.; Liu, J.; McClain, J. D.; Sayfutyarova, E. R.; Sharma, S.; Wouters, S.; Chan, G. K.-L. PySCF: The Python-Based Simulations of Chemistry Framework. WIREs Computational Molecular Science 2018, 8 (1), e1340.

(57) Sun, Q.; Zhang, X.; Banerjee, S.; Bao, P.; Barbry, M.; Blunt, N. S.; Bogdanov, N. A.; Booth, G. H.; Chen, J.; Cui, Z.-H.; Eriksen, J. J.; Gao, Y.; Guo, S.; Hermann, J.; Hermes, M. R.; Koh, K.; Koval, P.; Lehtola, S.; Li, Z.; Liu, J.; Mardirossian, N.; McClain, J. D.; Motta, M.; Mussard, B.; Pham, H. Q.; Pulkin, A.; Purwanto, W.; Robinson, P. J.; Ronca, E.; Sayfutyarova, E. R.; Scheurer, M.; Schurkus, H. F.; Smith, J. E. T.; Sun, C.; Sun, S.-N.; Upadhyay, S.; Wagner, L. K.; Wang, X.; White, A.; Whitfield, J. D.; Williamson, M. J.; Wouters, S.; Yang, J.; Yu, J. M.; Zhu, T.; Berkelbach, T. C.; Sharma, S.; Sokolov, A. Yu.; Chan, G. K.-L. Recent Developments in the PySCF Program Package. J. Chem. Phys. 2020, 153 (2), 024109. 